\title{
Review of the Agnostic-Type Treatment Approach: Treating Cancer by Mutations, Not by Location
}

\author{
Daniel Rosas · Luis E. Raez
}

Received: February 19, 2020 / Published online: May 2, 2020

(C) The Author(s) 2020

\begin{abstract}
Recent years has seen the discovery and description of genetic alterations responsible for oncogenesis in a wide variety of cancers, together with the finding that some markers are actionable and can be targeted by medications. Such developments have enabled cancer treatments to evolve from empirical palliative chemotherapy, with low chances of response or curative intent in most types of cancers, to targeted therapy, with some studies showing promising results in terms of improved overall response rates, overall survival and quality of life, although, like all new groups of medications, with specific adverse effect profiles. This treatment evolution is a major development in cancer therapy. Tumors were originally classified as solid or liquid tumors based on their location in the human body (solid organs or blood), which evolved into the medical
\end{abstract}

Digital Features To view digital features for this article go to https://doi.org/10.6084/m9.figshare.12129600.

D. Rosas $(\square)$

Internal Medicine, University of Texas Health

Science Center at San Antonio, 8639 Fairhaven

street apt 2431, San Antonio, TX 78229, USA

e-mail: rosas.daniel@icloud.com

\section{E. Raez}

Thoracic Oncology Program, Memorial Cancer Institute/Memorial Health Care System, Florida International University (FIU), Miami, FL, USA specialties of medical oncology and clinical hematology, respectively. Subsequently, tumors were classified by the organ they originate from, in the belief that the origin of the tumor would guide its biological behavior and would faciliate understanding of their mechanism of spread and, potentially, of the best treatment approach. Although this latter approach has achieved some success over the many years it has been applied, there have been major disappointments, particularly in lung cancers for which palliative chemotherapy has only been able to provide a median survival of around 1 year and a complete remission rate of $<5 \%$. We are now understanding that this concept of cancer pathophysiology is more complex, but also potentially simple, and that one or several molecular aberrations are probably responsible for the origin of each cancer. Various molecular alterations have been described, although the relevance of each alteration is not yet fully understood. In this article, we highlight clinical trial designs, biologic issues, and regulatory issues leading to the development of medications for tissue-agnostic treatment.

Keywords: Agnostic tumors; Mutations; Precision medicine; Translocations 


\section{Key Summary Points}

Treating tumors according to mutation and location has been increasing over the last decade and could possibly represent the future of oncology practice.

We have reviewed the most important mutations and the clinical data associated with these mutations.

\section{INTRODUCTION}

After years of palliative chemotherapy being the main therapy for non-small cell lung cancer (NSCLC), with a success of less than 5\% [1], more rational approaches to cancer care are finally being developed and established. Not only has it been discovered that molecular aberrations can be a target, but researchers have also identified the presence of these genetic aberrations in more than one tumor type. Ultimately, changes in molecular mechanisms, such as DNA mutations, translocations, deletions, fusions, and deficient DNA repair mechanisms, are responsible for the first step in the pathway leading to the origin and behavior of most cancers. This new knowledge is driving cancer therapies to change from treatments based on organ-directed therapy to those based on specific genetic alterations. The study of genetic alterations has recently become a focus of clinical studies, with the aim to provide different treatment approaches for a physician who can choose between different treatment options for patients. A new treatment option in oncology occurred when, for the first time ever, the anti-Programmed Death (PD)-1 immune checkpoint inhibitor pembrolizumab (KEYTRUDA ${ }^{\circledR}$; Merck \& Co.) was approved as the standard of care in patients with locally advanced or metastatic solid tumors with mismatch-repair deficiency (dMMR) or microsatellite instability-high (MSI-H) status who had progressed on first-line treatment and who had no other alternative treatment options [2]. The approval of this tissue-agnostic treatment will potentially change the future of the hematology-oncology field for the better.

Appication of a biomarker approach and focus on clinical development in an underserved patient population have demonstrated that this form of "personalized medicine" has clinical efficacy. This has led to the regulatory approval of medications for different types of tumors, even for some which can be used in both adult and pediatric patient populations [3]. In this article, we discuss the most common and significant genetic alterations, in our opinion, that are being studied and could be used in the tissue-agnostic treatment approach.

\section{Compliance with Ethics Guidelines}

This article does not contain any studies with human participants or animals performed by any of the authors.

\section{MICROSATELLITE INSTABILITY}

Microsatellite instability can arise from germline mutations or originate somatically. Immunotherapy has become an option for the treatment of almost all tumor types, especially after receiving approval from the U.S. Food and Drug Administration (FDA) for use in tumoragnostic treatment strategies [1]. This medication is for patients with solid tumors that have dMMR or MSI-H status whose disease has progressed on prior treatment and who have no alternative treatment options. It is also indicated in patients with colorectal cancer who have progressed on standard chemotherapy medications.

Tumors are classified as dMMR if they have somatic or germline mutations in the MMR genes (e.g., Lynch syndrome). MSI can also arise from epigenetic changes that altered microRNA pathways affecting MMR proteins [4]. MSI is most commonly found in endometrial and colon tumors, but it has also been detected in 24 cancer types, suggesting that MSI is a generalized target $[5,6]$. It is important to highlight that the genomes of dMMR tumors possess a 
high number of somatic mutations, making them more susceptible to immune checkpoint blockade regardless of tissue of origin.

In a large variety of tumors, immune checkpoint ligands on tumor cells interact with their immune cell receptors on effector T-cells, inhibiting the immune response that would attack the cancer cell. This interaction is blocked by the checkpoint inhibitors and leads to an immune cell activity response [7]; this immunologic activation can translate into a potent and durable anti-tumor effect. The identification of this immunologic activation/antitumor effect was a revolutionary scientific discovery, with the physicians working on and discovering this effect ultimately winning the Nobel Prize. The side effects of this encouraging new approach, however, are associated with new adverse effects. Although most patients develop only a non-serious rash, patients can suffer from life-threatening pneumonitis, hepatitis, colitis, or exacerbations of severe autoimmune disorders that had not been diagnosed earlier $[8,9]$. A strategy to enrich tumors to respond to checkpoint inhibition is an area under investigation. Predictive biomarkers include tumor mutation burden, RNA expression signature, PD-L1 expression, lymphocytic infiltrates, RNA expression signature, and mutation-associated neoantigens (MANAs) [10]. Studies on MANAs are ongoing, with some authors reporting that some dMMR cancers can be predicted to contain a large number of MANAs and hence be recognized by the immune system [11].

In a prospective study, Le et al. showed how pembrolizumab has a substantial benefit in dMMR cancers across different tumor types [12]. All patients enrolled in the study had received one prior therapy, had progressive disease, and had dMMR cancer assessed by either PCR or immunohistochemistry (IHC). Twelve cancer types were included. An objective radiographic response (ORR) was evidenced in $53 \%$ of patients, 21\% achieved a complete response (CR), and the disease control rate (DCR) was reached in $76 \%$ of patients. Neither median progression-free survival nor overall survival had been reached at the time of publication. Eleven patients achieved CR and were taken off therapy after 2 years on treatment; no evidence of cancer recurrence has been observed in these patients with a median time off therapy of 8.3 months. The adverse effects were easily manageable.

\section{RET FUSIONS}

Other genetic targets attracting increasing interest are the RET fusions, which to date have been a target with promising outcomes in lung, thyroid, and pancreatic cancers, among others. Although only a small subset of cancer types have this mutation, many of those that do, such as lung cancer, affect a large number of patients. Two medications, LOXO-292 and BLU-667 [4, 13], are currently being tested in ongoing phase III trials based on their efficacy in the LIBRETTO-001 trial of RET-fusion-positive NSCLC patients treated with LOXO-292 (selpercatinib; Eli Lilly and Company). The results of the LIBRETTO-001 trial were presented at the 2019 European Society for Medical Oncology (ESMO) and the 2019 Internal Association for the Study of Lung Cancer (IASLC) World Conference on Lung Cancer and Annual Meetings [4]. LOXO-292 was associated with significant activity in RET fusion-positive NSCLCs, showing an ORR of $68 \%$ in the primary analysis. Responses were durable, and LOXO-292 was overall well tolerated with mostly Grade 1-2 treatment-related adverse effects, with only a discontinuation rate of only $1.7 \%$. A similar activity was also reported in the RET mutant-activated medullary thyroid cancer (MTC). Based on these impressive results, a new drug application has been submitted to the FDA and two randomized phases III trials have been planned [13].

Although the data are not optimal to date, the data currently available demonstrate that the response rate is more than $50 \%$ in some types of genetic alterations, which brings hope for positive results in further studies.

\section{BRAFV600 MUTATION}

The BRAFv600 genetic aberration has been found in several solid tumors (e.g., melanoma, NSCLC, colon cancer) and in hematologic malignancies, such as hairy cell leukemia. The 
novel tyrosine kinase inhibitor (TKI) vemurafenib showed encouraging efficacy among melanoma patients enrolled in a phase 1 trial (81\% ORR). Subsequent identification of re-activation of the mitogen-activated protein kinase (MAPK) pathway as an escape mechanism led to the approval of a combination therapy of BRAF and mitogen-activated extracellular signal-related kinase (MEK) inhibitors for patients with BRAF-mutated melanoma [14, 15]. BRAF mutations were first shown to be involved in human cancers in 2002 [16], and they have subsequently been reported to be present in $7-15 \%$ of all cancers, of which the most common is melanoma (40-70\%) [17]. Other tumor types with a high prevalence of BRAFV600 mutations include multiple myeloma, hairy cell leukemia, papillary thyroid cancer, Erdheim-Chester disease, Langerhans cell histiocytosis, serous ovarian cancer, NSCLC, and colorectal cancer [18-20]. The BRAFV600 driver mutation is an oncogene that is often associated with an aggressive phenotype, with patients having a shorter disease-free survival and OS than those with wild-type BRAF cancers [21, 22]. Active therapies targeting this mutation in melanoma patients include the TKIs dabrafenib, vemurafenib, and encorafenib, and combinations of BRAF inhibitors and MEK inhibitors [23, 24]. In the most recent study of metastatic colorectal cancer, Kopetz et al. demonstrated how BRAFV600E inhibitors combined with anti-angiogenic and anti-MEK therapy after one or two lines of therapy achieved an OS higher than that of conventional therapy [25]. Thus, there is an increased research interest in the treatment of solid tumors with BRAFV600 mutations in cancers other than melanoma, such as lung cancers. The treatment of BRAF-mutated NSCLC with the combination of dabrafenib and the MEK inhibitor trametinib recently received FDA approval based on results from a trial that enrolled 93 patients who had received chemotherapy for advanced NSCLC [26]. In this study, the ORR was $62 \%$ and, interestingly, monotherapy with dabrafenib in 78 patients with BRAF-mutated NSCLC showed an ORR of $27 \%$. It is important to note that the addition of a MEK inhibitor (trametinib) was necessary to achieve a significantly higher response rate [26].
Cohn et al. published their results of a multicenter, national screening trial in 2017 for BRAFV600 mutations, which confirmed previously reported incidences of this driver oncogene $[27,28]$.

\section{NEUROTROPHIC TYROSINE RECEPTOR KINASE FUSION PROTEINS}

Neurotrophic tyrosine receptor kinase (NTRK) genes encode for the Trk family of tyrosine protein kinase receptors, namely, TrkA, TrkB, and TrkC (encoded by NTRK1, NTRK2, and NTRK3, respectably). These proteins are involved in the origin and development of the nervous system in utero and in the development of sensation pain, proprioception, and appetite postnatally under normal circumstances $[29,30]$. However, NTRK gene aberrations are also present in solid tumors as gene translocations or fusion proteins that are responsible for the abnormal cell proliferation. These oncogenic fusions are associated with poor overall survival rates in patients with lung and other types of cancer [31].

Kummar and Lassen published a review of NTRK gene alterations as one example of success in using the agnostic treatment approach. These NTRK fusions are common in rare cancers, such as infantile fibrosarcoma, and are rare in the more common cancers, such as gliomas, melanomas, thyroid cancer, breast cancer, and other adult epithelial cancers. Similar to observations reported on multiple oncogenes (e.g., ALK, BRAF, ROS1, and others), NTRK gene fusions are present in several different tumors (to date, 17 tumor types have been identified). These gene alterations are an example of why the tumor site-agnostic approach may be the future treatment approach in fighting cancer [32]. However, a number of challenges lie ahead before these fusion proteins can be diagnosed.

Clinical data pertaining to the treatment of NTRK cancers are currently available from phase I and phase II studies. Two medications, entrectenib and larotrectenib, have an acceptable side effect profile, with toxicities that are independent of tumor type, and they are 
Table 1 Objective response rates and overall survival across multiple studies for different tumor types

\begin{tabular}{llll}
\hline Study & Genetic aberration targeted & Objective response rate (\%) & Overall survival in months \\
\hline Le et al. [20] & MSI-H or MMR (+) & 53 & 5.0 \\
Dummer et al. [33] & BRAF & 64 & 33.6 \\
Dazhi Liu et al. [8] & NTRK & 86 & $-{ }^{\mathrm{a}}$ \\
Li et al. [32] & RET & 68 & 9.9 \\
Hauschild et al. [29] & BRAF & 50 & $-{ }^{\mathrm{b}}$ \\
Drilon et al. [7] & TRK & 80 & $-{ }^{\mathrm{a}}$ \\
Chapman et al. [30] & BRAFV600E & 48 & 84 \\
\hline
\end{tabular}

${ }^{a}$ Median overall survival has not yet been reached among all evaluable patients

b Too few deaths were reported for any conclusions to be drawn on overall survival

effective irregardless of patient age and fusion type $[33,34]$. Data on other agents in development, including LOXO 195, DS-6051b, TPX0005, ARRRY-470, and CEP-701, are also available $[33,34]$. It should be noted that although NTRK gene fusions are present in fewer than $1 \%$ of most cancers, in tumors such as lung cancer, this $1 \%$ becomes an important group of patients who would benefit from this agnostic treatment approach.

Techniques used currently for the molecular diagnosis of these aberrations include, among others, next-generation sequencing (NGS), IHC, fluorescence in situ hybridization (FISH), and reverse-transcriptase PCR (RT-PCR) of DNA, RNA, or cell-free DNA (cfDNA).

All of these techniques are associated with specific benefits and limitations. It is also important to mention that in many instances there are insufficient tumor tissue specimens for testing. For example, the recommendation for lung cancer is to test for genetic alterations, specifically for ALK and ROS1 translocations, with the FISH method; however, recent data indicates that more FISH tests will be needed to test for the NTRKs (and perhaps also for one or more RET fusions). This clearly increases the cost of the workup for these patients while providing more individual-based care. In addition, the gene fusion partner, which may be important for treatment or as a mutation burden, might not be identified by these techniques. Another technique, RT-PCR, is very sensitive, but this would need a high number of primers to cover all known NTRK aberrations because there are more than 60 NTRK fusions documented to date; thus, the economic burden becomes an issue. The question which needs to be asked is: Is the solution to this issue to develop improved IHC methods, like we are currently doing for ALK and hopefully will one day have for the ROS1 and NTRK oncogenes? Or should we try establishing NGS as the standard of care, followed by a more specific follow-up-for example, by looking for TKI-resistant ALK variants? For those who question NGS, we can say that at the moment several NTRK 1-3 introns are not covered by available NGS panels, so the most up-to-date scientific methods still have their limitations. Alternatively, should we do RNA transcriptome sequencing as a complement of the DNA? Or should a whole-genome NGS be performed to find all possible alterations while significantly increasing the cost of the workup?

These are some of the questions that remain while there is a need to increase the accuracy of available techniques to make them more costeffective. Hopefully in the near future, upcoming treatment guidelines can adapt this technique to cover a larger variety of types of tumors.

Table 1 shows the very high response rates (ORR) and OS that are achieved due to this type of personalized medicine. It should be noted that these values are two- to threefold those expected with palliative chemotherapy. 
In one study with BLU-667, two patients with MTC showed a $>90 \%$ reduction in serum calcitonin and a $57-75 \%$ in the tumor marker carcinoembryonic antigen (CEA) together with a $19 \%$ reduction in target lesion size after 8 weeks of treatment, and two patients with NSCLC who received BLU-667 achieved a 25-34\% reduction in tumor size followed by Partial Remission [13].

\section{CONCLUSION}

In this article we have stated that several actionable somatic cancer genetic aberrations (NTRK, BRAF, MSI-H, dMMR, RET) are present in a wide variety of different types of tumors, supporting the concept of tissue-agnostic tumor therapy. The organ-based clinical models that we had been using to guide therapy are not adequate to predict tumor response or resistance and need to evolve to allow healtcare professionals to carry out precision and personalized medicine. A new generation of oncology trials of tumor type-agnostic medications bring hope that new pathways of medication discovery and development will be found and that these will bring us one step closer to winning the fight against cancer.

\section{ACKNOWLEDGEMENTS}

Funding. No external funding was used in the preparation of this manuscript.

Authorship. All named authors meet the International Committee of Medical Journal Editors (ICMJE) criteria for authorship for this article, take responsibility for the integrity of the work as a whole, and have given their approval for this version to be published.

Disclosures. Luis E. Raez has research support from LOXO, Genentech. Lilly Oncology and Merck oncology. Daniel Rosas has nothing to disclose.
Compliance with Ethics Guidelines. This article does not contain any studies with human participants or animals performed by any of the authors.

Data Availability. Data sharing is not applicable to this article as no datasets were generated or analyzed during the current study.

Open Access. This article is licensed under a Creative Commons Attribution-NonCommercial 4.0 International License, which permits any non-commercial use, sharing, adaptation, distribution and reproduction in any medium or format, as long as you give appropriate credit to the original author(s) and the source, provide a link to the Creative Commons licence, and indicate if changes were made. The images or other third party material in this article are included in the article's Creative Commons licence, unless indicated otherwise in a credit line to the material. If material is not included in the article's Creative Commons licence and your intended use is not permitted by statutory regulation or exceeds the permitted use, you will need to obtain permission directly from the copyright holder. To view a copy of this licence, visit http://creativecommons.org/licenses/by$\mathrm{nc} / 4.0 /$.

\section{REFERENCES}

1. Schiller JH, Harrington D, Belani CP, et al. Comparison of four chemotherapy regimens for advanced non-small-cell lung cancer. N Engl J Med. 2002;346(2):92-8.

2. U.S. Food and Drug Administration. FDA grants accelerated approval to pembrolizumab for first tissue/site agnostic indication. https://www.fda. gov/Drugs/InformationOnDrugs/ApprovedDrugs/u cm560040.htm. Accessed 11 Apr 2019.

3. Yan L, Zhang W. Precision medicine becomes reality-tumor type-agnostic therapy. Cancer Commun. 2018;38(1):6.

4. National Institutes of Health (NIH) ClinicalTrials.gov. A study to test the safety of the investigational drug selitrectinib in children and adults that may treat cancer. https://www.clinicaltrials.gov/ 
ct2/show/NCT03215511?cond=LOXO-195\&rank= 1. Accessed 11 Apr 2019.

5. Murphy KM, Zhang S, Geiger T, et al. Comparison of the microsatellite instability analysis system and the Bethesda panel for the determination of microsatellite in-stability in colorectal cancers. J Mol Diagn. 2006;8:305-11.

6. Zehir A, Benayed R, Shah RH, et al. Mutational landscape of metastatic cancer revealed from prospective clinical sequencing of 10,000 patients. Nat Med. 2017;23:703-13.

7. Le DT, Durham JN, Smith KN, et al. Mismatch repair deficiency predicts response of solid tumors to PD-1 blockade. Science. 2017;357:409-13.

8. Topalian SL, Sznol M, McDermott DF, et al. Survival, durable tumor remission, and long-term safety in patients with advanced melanoma receiving nivolumab. J Clin Oncol. 2014;32:1020-30.

9. Gettinger SN, Horn L, Gandhi L, et al. Overall survival and long-term safety of nivolumab (anti-programmed death 1 antibody, BMS-936558, ONO4538 ) in patients with previously treated advanced non-small-cell lung cancer. J Clin Oncol. 2015;33: 2004-122.

10. Ward JP, Gubin MM, Schreiber RD. The role of neoantigens in naturally occurring and therapeutically induced immune responses to cancer. Adv Immunol. 2016;130:25-74.

11. Smyrk TC, Watson P, Kaul K, Lynch HT. Tumorinfiltrating lymphocytes are a marker for microsatellite instability in colorectal carcinoma. Cancer. 2001;91:2417-22.

12. Le DT, Uram JN, Wang H, et al. PD-1 Blockade in tumors with mismatch-repair deficiency. N Engl J Med. 2015;372(26):2509-20.

13. Subbiah V, Gainor JF, Rahal R, et al. Precision targeted therapy with BLU-667 for RET-driven cancers. Cancer Discov. 2018;8:836-49. https://doi.org/10. 1158/2159-8290.CD-18-0338.

14. Li AY, McCusker MG, Russo A, et al. RET fusions in solid tumors. Cancer Treat Rev. 2019;81:101911.

15. Davies H, Bignell GR, Cox C, et al. Mutations of the BRAF gene in human cancer. Nature. 2012;417(6892):949-54.

16. Dhomen N, Marais R. New insight into BRAF mutations in cancer. Curr Opin Genet Dev. 2007;17(1):31-9.

17. Tiacci E, Schiavoni G, Forconi F, et al. Simple genetic diagnosis of hairy cell leukemia by sensitive detection of the BRAF-V600E mutation. Blood. 2012;119(1):192-5.

18. Grisham RN, Iyer G, Garg K, et al. BRAF mutation is associated with early stage disease and improved outcome in patients with low-grade serous ovarian cancer. Cancer. 2013;119(3):548-54.

19. Xi L, Arons E, Navarro W, et al. Both variant and IGHV4-34-expressing hairy cell leukemia lack the BRAF V600E mutation. Blood. 2012;119(14): 3330-2.

20. Marchetti A, Felicioni L, Malatesta S, et al. Clinical features and outcome of patients with non-smallcell lung cancer harboring BRAF mutations. J Clin Oncol. 2011;29(26):3574-9.

21. Xing M, Alzahrani AS, Carson KA, et al. Association between BRAF V600E mutation and mortality in patients with papillary thyroid cancer. JAMA. 2013;309(14):1493-501.

22. Roth AD, Tejpar S, Delorenzi M, et al. Prognostic role of KRAS and BRAF in stage II and III resected colon cancer: results of the translational study on the PETACC-3, EORTC 40993, and SAKK 60-00 trial. J Clin Oncol. 2010;28(3):466-74.

23. Hauschild A, Grob J-J, Demidov LV, et al. Dabrafenib in BRAF-mutated metastatic melanoma: a multicentre, open-label, phase 3 randomized controlled trial. Lancet. 2012;380(9839):358-65.

24. Chapman PB, Hauschild A, Robert C, et al. Improved survival with vemurafenib in melanoma with BRAF V600E mutation. $\mathrm{N}$ Engl J Med. 2011;364(26):2507-16.

25. Dummer R, Ascierto PA, Gogas HJ, Aet al. Encorafenib plus binimetinib versus vemurafenib or encorafenib in patients with BRAF-mutant melanoma (COLUMBUS): a multicentre, open-label, randomized phase 3 trial. Lancet Oncol. 2018;19(5): 603-15.

26. A study of repotrectinib (TPX-0005) in patients with advanced solid tumors harboring ALK, ROS1, or NTRK1-3 Rearrangements- ClinicalTrials.gov. https://www.clinicaltrials.gov/ct2/show/NCT03093 116? cond=TPX-0005\&rank=1. Accessed 24 Apr 2020.

27. A phase I/IIa open-label, dose escalation and cohort expansion trial of oral TSR-011 in patients with advanced solid tumors and lymphomas - Full Text View - ClinicalTrials.gov. https://www.clinicaltrials. gov/ct2/show/NCT02048488?cond=TSR-011. Acce ssed 24 Apr 2020.

28. Phase 1 Study of DS-6051b in Japanese Subjects With Advanced Solid Malignant Tumors- 
ClinicalTrials.gov. https://www.clinicaltrials.gov/ ct2/show/NCT02675491?cond=DS-6051b. Accessed 24 Apr 2020.

29. Brodeur GM, Minturn JE, Ho R, et al. Trk receptor expression and inhibition in neuroblastomas. Clin Cancer Res. 2009;15:3244-50.

30. Okamura K, Harada T, Wang S, et al. Expression of TrkB and BDNF is associated with poor prognosis in non-small cell lung cancer. Lung Cancer. 2012;78: 100-6.

31. Drilon A, Laetsch TW, Kummar S, et al. Efficacy of Larotrectinib in TRK fusion-positive cancers in adults and children. N Engl J Med. 2018;378(8): 731-9.
32. Liu D, Offin M, Harnicar S, Li BT, Drilon A. Entrectinib: an orally available, selective tyrosine kinase inhibitor for the treatment of NTRK, ROS1, and ALK fusion-positive solid tumors. TCRM. 2018;14:1247-52.

33. Ardini E, Bosotti R, Borgia AL, De Ponti C, Somaschini A, Cammarota R, et al. The TPM3-NTRK1 rearrangement is a recurring event in colorectal carcinoma and is associated with tumor sensitivity to TRKA kinase inhibition. Mol Oncol. 2014;8: 1495-507.

34. Kummar S, Lassen U. TRK inhibition-a new tumor-agnostic treatment strategy. Targ Oncol. 2018. https://doi.org/10.1007/s11523-018-0590-1. 